\title{
Platelets mediate lymphovenous hemostasis to maintain blood-lymphatic separation throughout life
}

\author{
Paul R. Hess, ${ }^{1}$ David R. Rawnsley, ${ }^{1}$ Zoltán Jakus, ${ }^{1}$ Yiqing Yang, ${ }^{1}$ Daniel T. Sweet, ${ }^{1}$ Jianxin Fu, ${ }^{2}$ \\ Brett Herzog, ${ }^{2}$ MinMin Lu, ${ }^{1}$ Bernhard Nieswandt, ${ }^{3}$ Guillermo Oliver, ${ }^{4}$ Taija Makinen, ${ }^{5}$ \\ Lijun Xia, ${ }^{2}$ and Mark L. Kahn'
}

\begin{abstract}
1Department of Medicine and Division of Cardiology, University of Pennsylvania, Philadelphia, Pennsylvania, USA. ${ }^{2}$ Cardiovascular Biology Research Program, Oklahoma Medical Research Foundation, Oklahoma City, Oklahoma, USA. ${ }^{3}$ University Hospital Würzburg and Rudolf Virchow Center, DFG-Research Center for Experimental Biomedicine, Würzburg, Germany. “Department of Genetics, St. Jude Children's Research Hospital, Memphis, Tennessee, USA. ${ }^{5}$ Lymphatic Development Laboratory, Cancer Research UK London Research Institute, London, England.
\end{abstract}

\begin{abstract}
Mammals transport blood through a high-pressure, closed vascular network and lymph through a lowpressure, open vascular network. These vascular networks connect at the lymphovenous (LV) junction, where lymph drains into blood and an LV valve (LVV) prevents backflow of blood into lymphatic vessels. Here we describe an essential role for platelets in preventing blood from entering the lymphatic system at the LV junction. Loss of CLEC2, a receptor that activates platelets in response to lymphatic endothelial cells, resulted in backfilling of the lymphatic network with blood from the thoracic duct (TD) in both neonatal and mature mice. Fibrin-containing platelet thrombi were observed at the LVV and in the terminal TD in wild-type mice, but not Clec2-deficient mice. Analysis of mice lacking LVVs or lymphatic valves revealed that platelet-mediated thrombus formation limits LV backflow under conditions of impaired valve function. Examination of mice lacking integrin-mediated platelet aggregation indicated that platelet aggregation stabilizes thrombi that form in the lymphatic vascular environment to prevent retrograde blood flow. Collectively, these studies unveil a newly recognized form of hemostasis that functions with the LVV to safeguard the lymphatic vascular network throughout life.
\end{abstract}

\section{Introduction}

The mammalian cardiovascular system is divided into distinct blood and lymphatic vascular networks: blood is transported to the tissues in a closed, high-pressure vascular system, and interstitial fluid is transported from the tissues in an open, low-pressure vascular system (1). These structurally and functionally distinct vascular networks must remain separate to function properly, yet they must also connect in order for lymph fluid and immune cells to be returned to the blood. A bicuspid lymphovenous valve (LVV) is believed to safeguard this connection and prevent blood from entering the lower-pressure lymphatic system and blocking forward lymphatic flow. In humans, LVVs reside in 2 locations: the thoracic duct-subclavian vein (TD-SV) junction on the left, and the right lymphatic duct-SV junction on the right (2).

Recent genetic studies in mice have revealed an unexpected mechanism, in which platelet C-type lectin-like receptor 2 (CLEC2) signaling in response to the surface protein podoplanin (PDPN) expressed on lymphatic endothelial cells (LECs) is required for normal lymphatic development and function. Mice deficient in PDPN, CLEC2, or the CLEC2 signaling proteins SYK and SLP-76 exhibit blood-filled lymphatics during fetal life and die shortly after birth due to defective lymphatic function (3-6). Tissue-specific deletion experiments reveal that CLEC2 and its intracellular signaling effectors are required in platelets, while PDPN is required in endothelial cells to prevent this phenotype (4-7). In addition, studies of mature mice reconstituted with hematopoietic cells deficient in Clec2,

Conflict of interest: The authors have declared that no conflict of interest exists. Citation for this article: J Clin Invest. 2014;124(1):273-284. doi:10.1172/JCI70422.
Syk, or SLP-76, as well as mature mice in which PDPN expression is ablated through conditional deletion of the O-glycan synthase enzyme required for its expression, revealed that this pathway is also required in mature animals to prevent blood-filled lymphatics and death due to lymphatic dysfunction $(3,4,8,9)$.

How platelet activation by LECs maintains separation of the blood and lymphatic vascular networks has not been clear. Some groups have reported that platelet activation by CLEC2 signaling may regulate LEC growth in vitro through secretion of platelet granule contents $(6,10)$. However, we have not observed such effects on LEC growth in vitro or detected major defects in the pattern or rate of lymphatic vascular development in vivo $(8,11)$, and how such an angiogenic mechanism would explain an ongoing requirement in mature, presumably nonangiogenic, animals is not clear. Understanding the role of platelet CLEC2 signaling in lymphatic growth and function has acquired particular urgency with the recent development and clinical application of therapeutic agents that block SYK, a nonreceptor tyrosine kinase that is required for CLEC2 signaling in platelets.

A major hurdle in understanding this pathway has been defining precisely when and where platelets in circulating blood come into contact with LECs to initiate PDPN-CLEC2 signaling. We and others have identified platelets in contact with newly specified LECs in the cardinal vein of E11.5 mouse embryos $(4,12)$, but venous LEC specification is complete by E14.5 and cannot explain the requirement for this pathway in older embryos and mature animals. In the present study, we used anti-CLEC 2 antibodies that rapidly induce CLEC2 deficiency in vivo to determine the requisite sites of platelet-LEC interaction. Our studies revealed that plate- 
lets interact with lymphatic endothelium specifically at the TD-SV junction and that blood-filled lymphatics arise due to backfilling of the lymphatic vascular network from this site. Identical findings were observed in fully mature animals after reconstitution with $\mathrm{Clec} 2^{-/-}$hematopoietic cells, which indicates that this hemostatic requirement exists throughout life, even in animals with entirely normal lymphatic development and function. Analysis of mice lacking LVVs or lymphatic valves revealed that valves and platelet-mediated lymphovenous (LV) hemostasis function together to maintain blood-lymphatic separation. These results identify an unexpected role for platelets in mediating a specialized form of intervascular hemostasis that differs markedly from the bettercharacterized role of platelets in high shear, arterial hemostasis, and thrombosis. Our findings raise important questions regarding the role of this LV hemostatic pathway in pathologic states and in patients treated with drugs that block platelet function.

\section{Results}

Anti-CLEC2 antibodies rapidly confer blood-filled lymphatics in the intestine of postnatal animals. Loss of CLEC2 signaling in late-gestation embryos and in mature animals reconstituted with $\mathrm{Clec} 2^{-/-}$hematopoietic cells resulted in blood-filled lymphatics throughout the intestine (Figure 1, A and B). Clec2 ${ }^{-/-}$postnatal animals also exhibited large pleural effusions that were associated with respiratory distress and death (Figure 1C). To define the sites of platelet-LEC interaction at which PDPN-CLEC2 signaling is required to prevent this phenotype, we performed extensive histologic staining for platelets and LECs in the intestine of wild-type late-gestation embryos and neonates. In contrast to a similar histologic analysis that revealed platelet-LEC interactions in the cardinal vein of the E11.5 embryo (4), the present studies failed to reveal any sites at which platelets associated with LECs $(N>100$ sections stained). These findings suggest either that histologic analysis is not sensitive enough to identify important sites of platelet-LEC interaction within the intestine or that blood-lymphatic mixing in the intestine might arise due to loss of platelet-LEC interaction at a distant site.

To define where platelet-LEC interaction is necessary, we adopted an anatomic strategy in which we rapidly induced a CLEC2 deficiency state in neonates and searched for the earliest sites of blood appearance in the lymphatics. CLEC2 deficiency was induced by injection of the rat anti-mouse CLEC 2 antibody INU1 in wild-type neonates. INU1 injection in mature animals has previously been shown to result in a transient thrombocytopenia that is followed by an extended period of complete platelet CLEC2 deficiency (13). Consistent with these findings, injection of INU1 induced a rapid and virtually complete loss of platelet CLEC2 receptors in neonatal mice (Figure 1D). This was a CLEC2-specific effect, as the levels of the platelet receptor GP1b were unchanged (Figure 1D). INU1injected neonates developed blood-filled lymphatics in the intestine and mesentery identical to those observed in Clec2-/- neonates as soon as 4 days after antibody administration (Figure 1E). These studies confirmed that CLEC2 signaling is required to prevent blood from entering the lymphatic network after birth and defined a means by which to trace the sites where platelet-LEC interaction is required to prevent this phenotype in postnatal animals.

Blood enters the intestine from mesenteric lymphatics in $\mathrm{Clec}^{-{ }^{--}}$animals. Lymphatic flow in the intestine begins with villous lacteals that connect to submucosal lymphatics and subsequently drain into mesenteric collecting lymphatics. Mesenteric collecting lymphatics drain through mesenteric LNs and merge at the cisterna chyli to form the TD. To determine the sites of origin of blood in the intestinal lymphatics of Clec $2^{-/-}$mice, we examined animals between 4 and 6 days after INU1 injection, the period of time during which the first blood-filled vessels were observed (Figure 1E). This spatiotemporal analysis revealed 3 distinct patterns of bloodfilled lymphatics in the abdomen of live animals. In the first, blood was only observed in mesenteric LNs, but not in lymphatic vessels of the mesentery or the intestine (Figure 2, A and B). In the second, blood was observed in both mesenteric LNs and mesenteric lymphatics, but not in lymphatic vessels of the intestine (Figure 2C). In the third, blood was observed at all 3 sites (Figure 2D). These patterns of blood-filled lymphatics were consistent with a previous study that described variable degrees of blood-filled lymphatics in the intestine and mesentery of mature mice reconstituted with SLP-76-deficient hematopoietic cells, with the mildest being the presence of blood-filled mesenteric LNs (14). These unexpected findings suggested that blood enters the lymphatics of the intestine from mesenteric lymphatics, rather than vice versa, in the absence of CLEC2 function. Thus, blood-filled lymphatics in the intestine of mice lacking PDPN-CLEC2 signaling are not the consequence of a primary vascular defect in the intestine itself, but instead arise secondarily as blood fills mesenteric lymphatics.

Blood-filled lymphatics arise in Clec2-/- animals lacking LNs. LNs develop in association with lymphatic vessels, contain closely apposed blood and lymphatic vascular networks, and are bloodfilled throughout the lifetime of mice lacking PDPN-CLEC2 signaling $(8,14)$. We therefore hypothesized that the blood observed in mesenteric lymphatics shortly after loss of CLEC2 might originate in mesenteric LNs. To test this possibility, we examined Clec2 deficiency in mice that lack the cytokine LTA or the transcription factor RORC (also known as RORgt). $\mathrm{Lta}^{-/-}$and $\mathrm{Rorc}^{-/-}$animals failed to form LNs and other secondary lymphoid organs, such as Peyer's patches, due to an early defect in secondary lymphoid organ development (Figure 2E and refs. 15, 16). $\mathrm{Lta}^{-/-} \mathrm{Clec}^{-{ }^{--}}$and Rorc $^{-1-} \mathrm{Clec}^{-/-}$neonates exhibited blood-filled intestinal and mesenteric lymphatics like those observed in $\mathrm{Clec}^{-{ }^{--}}$neonates (Figure 2F). INU1 injection of $\mathrm{Lta}^{-/-}$and $\mathrm{Rorc}^{-/-}$neonates resulted in bloodfilled intestinal and mesenteric lymphatics in a pattern similar to those observed in wild-type animals (Figure 2G). Finally, bloodfilled intestinal and mesenteric lymphatics were also observed in lethally irradiated $\mathrm{Lta}^{-/-}$and $\mathrm{Rorc}^{-/-}$adult animals that were reconstituted with Clec2 ${ }^{-/-}$hematopoietic cells (Figure $2 \mathrm{H}$ ). These pharmacologic and genetic studies demonstrated that LNs are not required for the development of blood-filled mesenteric lymphatic vessels in perinatal or mature Clec2 $2^{-/-}$animals (Figure 2I).

The LV junction is the site of blood entry into the lymphatic network after loss of CLEC2. The finding that blood entered the intestine from mesenteric lymphatic vessels in a manner independent of mesenteric LNs after CLEC2 loss suggested that the critical site of platelet interaction must lie in the most terminal portion of the lymphatic network, most likely the TD (Figure 3A). To test this hypothesis, we examined the TD at early time points after INU1induced CLEC2 deficiency. The TD was readily visualized by the presence of chyle or by the presence of fluorescent dextran after injection into the footpad (Figure 3B), which indicates that forward lymphatic flow is present in some animals that exhibit blood in the lymphatic network. Analysis of INU1-injected neonates at earlier time points revealed blood in the TD in the chest prior to detection of blood in mesenteric LNs or mesenteric-collecting lymphatics in the abdomen (Figure 3C). In addition, blood was 
A
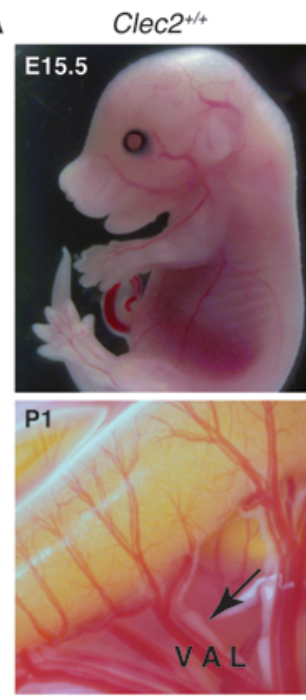

C
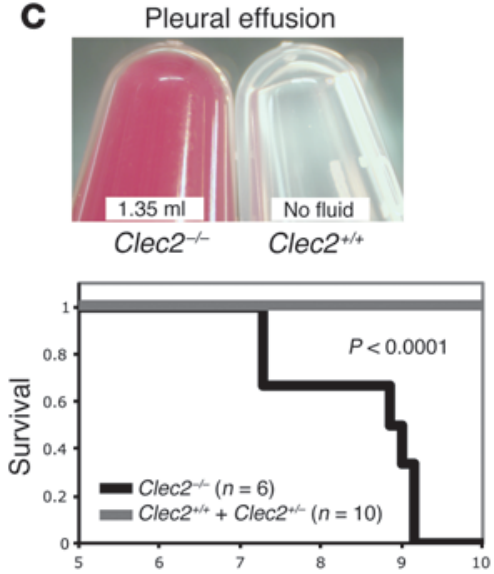

$\mathbf{E}$
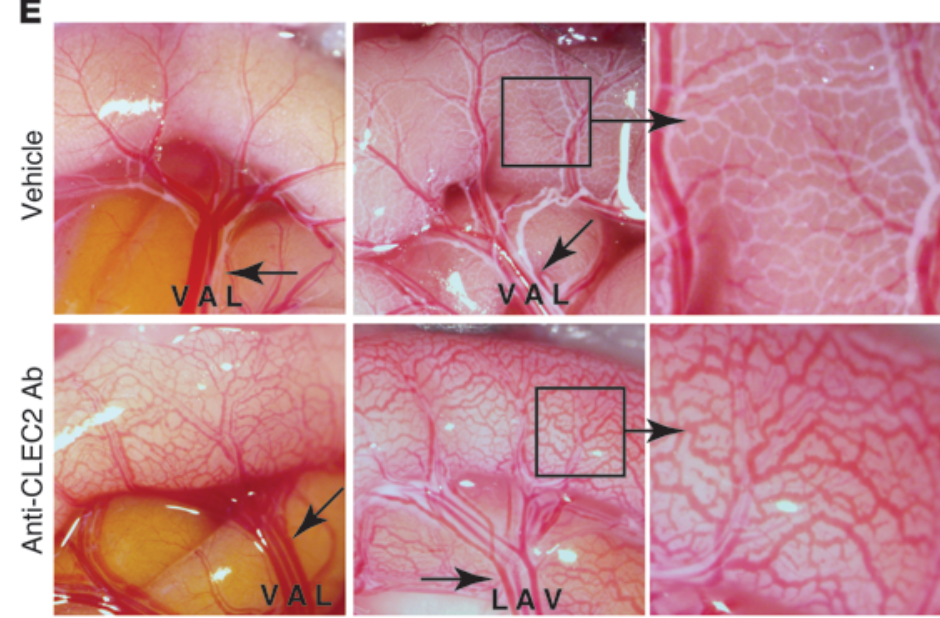

B
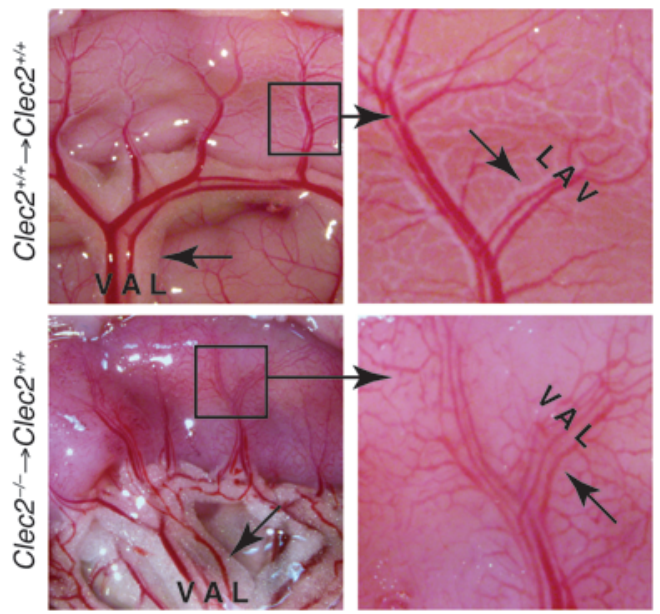

Neonatal platelets
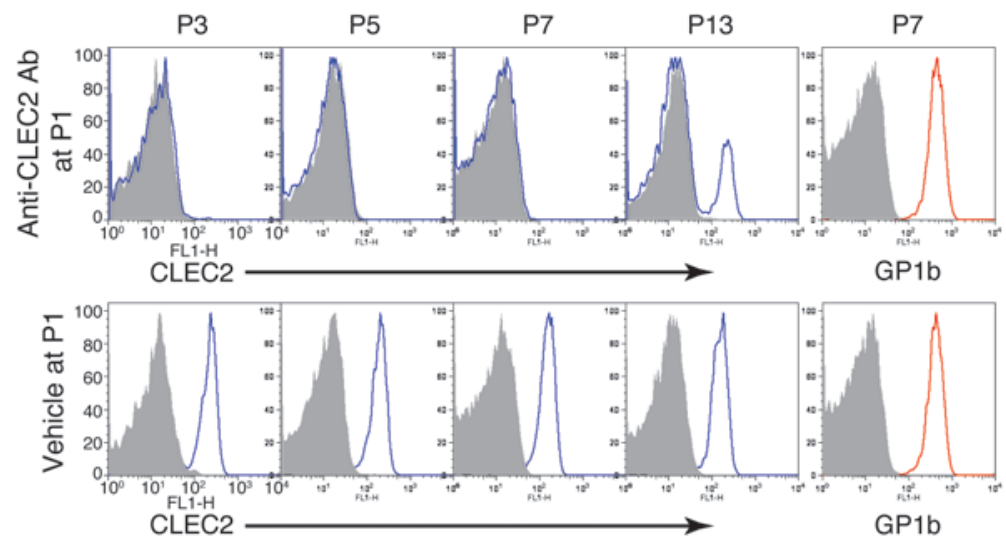

Figure 1

Loss of CLEC2 results in blood-filled lymphatics in the intestine of both perinatal and mature mice. (A) Genetic deletion of Clec2 resulted in blood-filled lymphatics in the skin at midgestation (top) and in the small intestine at birth (bottom). (B and C) Mature wild-type mice reconstituted with $\mathrm{Clec}^{-1-}$ hematopoietic cells exhibited blood-filled intestinal lymphatics (B) and large, bloody pleural effusions (C). (B) Images of the intestine were obtained 5.5 weeks after transplantation of the indicated hematopoietic cells. Respiratory distress and death were observed in animals with $\mathrm{Clec2} 2^{-/-}$, but not $\mathrm{Clec}^{+/+}$or Clec2 ${ }^{+/-}$, hematopoietic cells. (C) Pleural effusion fluid was obtained at the time of sacrifice. The survival curve of a transplantation cohort is also shown. A large pleural

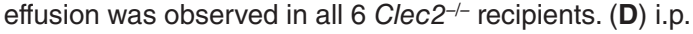
injection of the anti-CLEC2 antibody INU1 at P1 resulted in a sustained CLEC2 deficiency state. Shown is flow cytometry of circulating platelets stained with anti-CLEC2 or anti-GP1b antibodies. (E) INU1-mediated CLEC2 deficiency conferred blood-filled intestinal lymphatics. Images of intestine at P5, after a single INU1 injection at P1 (left), or at P14, after injection at P1, P5, and P9 (middle and right). Arrows indicate lymphatic vessels. Boxed regions are shown at higher magnification (enlarged $\times 3$ ). V, vein; A, artery, L, lymphatic. 
A
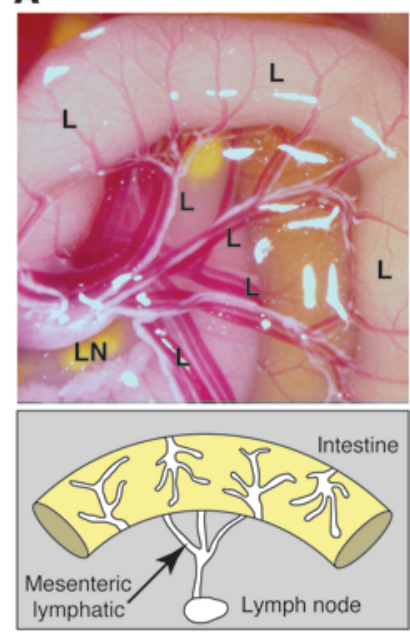

B
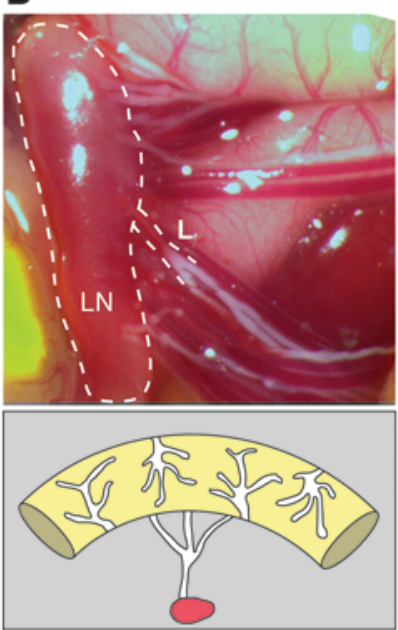

C
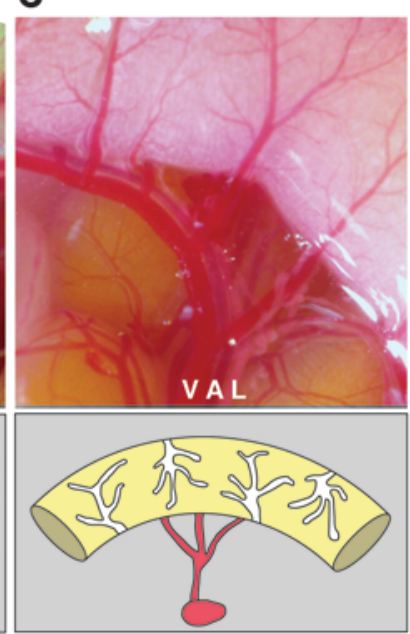

D

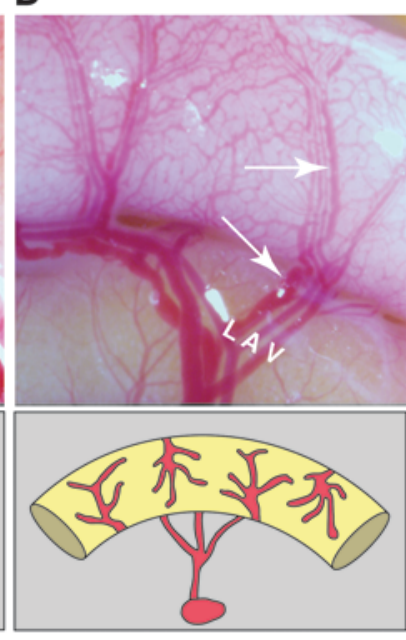

Anti-CLEC2 Ab
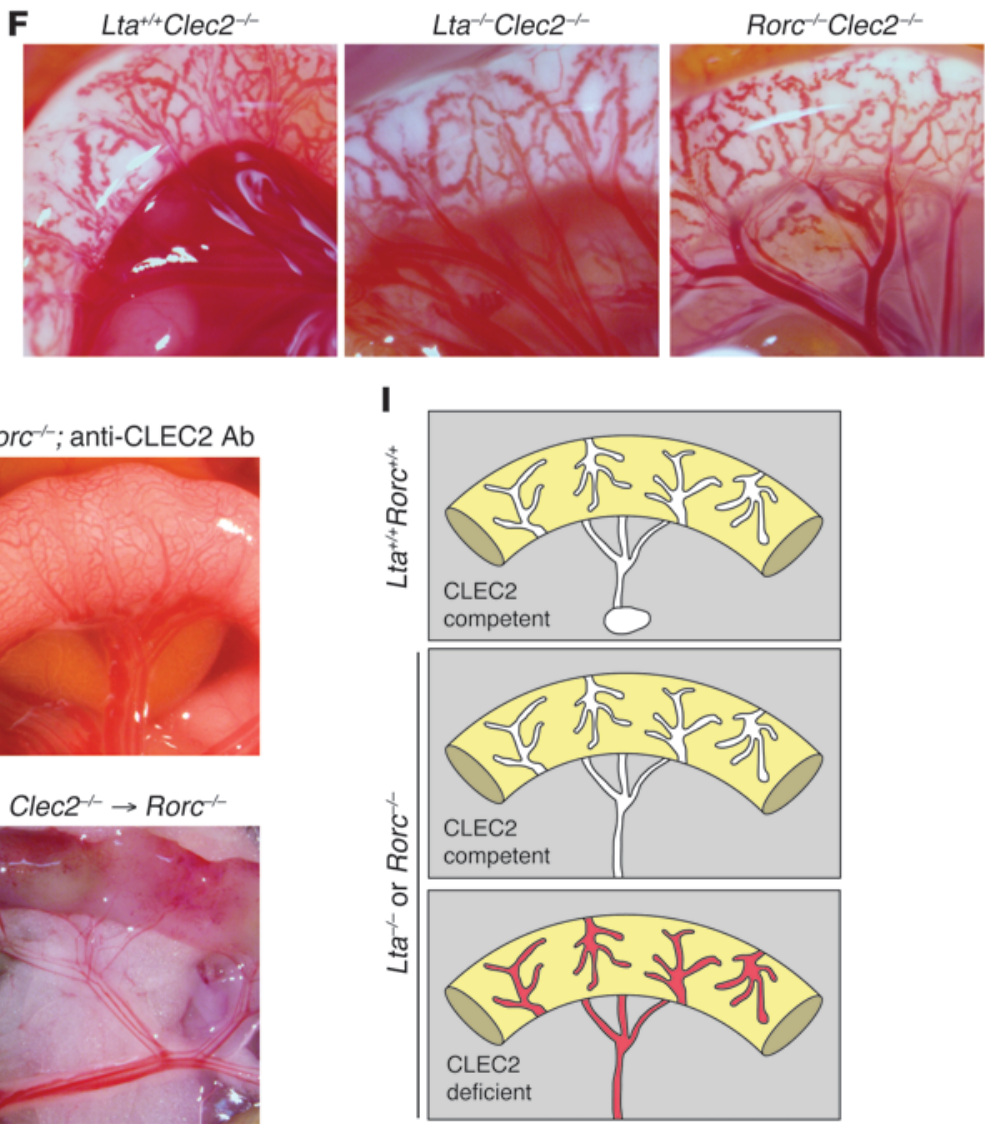

\section{Figure 2}

Blood enters lymphatic vessels of the intestine via backflow from mesenteric-collecting lymphatics in Clec2 $2^{-/-}$animals. (A) Normal anatomy of the mesentery and intestine at P6. Lymphatic vessels of the intestine and mesentery are white due to the presence of chyle. Shown are a mesenteric LN and a schematic of the lymphatic network at this site. (B-D) 3 patterns of blood-filled lymphatics were observed between P5 and P7 after anti-CLEC2 antibody injection at P1: (B) blood only in mesenteric LNs; (C) blood in mesenteric LNs and mesenteric lymphatic vessels, but not intestinal lymphatics; and (D) blood in mesenteric LNs, mesenteric lymphatics, and intestinal lymphatics. Dotted outline in B denotes LN; arrows in D indicate lymphatic vessels. (E) $\mathrm{Lta}^{-/-}$neonates lacked mesenteric LNs. Images were obtained at P1. (F) Clec2 $2^{-/-}$animals developed blood-filled lymphatics in the intestine on $\mathrm{Lta}^{-/-}$and $\mathrm{Rorc}^{-/-}$backgrounds. Images were obtained at P1. (G) $L t a^{-/-}$and Rorc ${ }^{-/-}$neonates developed blood-filled lymphatics after injection of anti-CLEC2 antibody. Antibody was injected at P1 and P5, and images were obtained at P9. (H) Lta ${ }^{-/-}$and Rorc $^{-/}$mature animals developed blood-filled lymphatics after reconstitution with $\mathrm{Clec}^{-/-}$hematopoietic cells. Images were obtained 8 weeks after transplantation. (I) Lta or Rorc deficiency does not prevent blood from entering the intestines of $\mathrm{Clec}^{-/-}$animals. 
observed in the most terminal portion of the TD - immediately preceding the TD-SV junction - when no blood was present in more proximal portions of the TD closer to the intestine (Figure $3 \mathrm{C})$. These findings suggest that blood enters the lymphatic system at the LV junction, where the TD joins the SV, and that the intestine and other lymphatic vascular beds are filled secondarily by retrograde blood flow.

Aspects of lymphatic growth such as valve maturation are believed to take place and/or be reversible in the perinatal period (17). It is therefore possible that retrograde entry of blood into the terminal TD in Clec2 $2^{-/}$neonates could arise secondary to effects of blood-filled lymphatics on valve development and maturation, and not reflect a primary hemostatic requirement in the presence of normal LVVs and lymphatic valves. To distinguish between these possibilities, we performed similar-timed CLEC2 deficiency experiments in mature 12-week-old wild-type animals that were lethally irradiated and reconstituted with Clec2 $2^{-/}$hematopoietic cells. Analysis of these animals 3 weeks after reconstitution revealed the presence of blood in the TD prior to its detection in mesenteric LNs, mesenteric collecting lymphatics, or lymphatics in the intestine wall (Figure 3D). These findings demonstrated that CLEC2-mediated platelet activation is required in healthy animals to prevent retrograde filling of the lymphatic system with venous blood even after normal LVV and lymphatic valve development (Figure 3E).

SYK inhibition with fostamatinib disodium (R788) does not result in entry of blood into the lymphatic network. Genetic studies indicate that SYK is absolutely required for CLEC2 signaling in platelets (6), and Syk-deficient neonates and adult radiation chimeras exhibit lethal blood-lymphatic vascular mixing phenotypes identical to those of Clec2-deficient animals $(9,18,19)$. It is therefore possible that novel SYK inhibitors developed to treat chronic inflammatory disorders such as rheumatoid arthritis may confer blood-lymphatic phenotypes like those associated with lymphatic dysfunction and death in genetic models and anti-CLEC2 antibody treatment. To test the effect of such inhibitors, we treated wild-type neonates for 8 days with the SYK antagonist fostamatinib disodium (R788) at doses higher than those shown to be effective in alleviating symptoms of experimental arthritis in a rat model (20). Fostamatinib is a prodrug that is rapidly converted to R406, with a plasma halflife of less than 2 hours in mice (21). In contrast, R788 has a halflife of approximately 15 hours in humans, where SYK inhibition is more sustained (22). Therefore, we administered fostamatinib every 6 hours to more accurately model human use. Unlike treatment with the antibody INU1 (Figure 3C), fostamatinib treatment did not result in detectable entry of blood into the TD $(n=6$; Figure $3 \mathrm{~F}$ ). As discussed further below, these findings suggest that very-high-level loss of SYK or CLEC2 function is required to confer blood-lymphatic mixing in healthy animals.

Platelet thrombiform at the LV junction in wild-type mice, but not Clec2-1mice. Bicuspid valves at the LV junction are thought to prevent venous blood from entering the lymphatic vascular network (Figure $4 \mathrm{~A}$ and ref. 2), but the studies described above suggested that platelet-LEC interaction and CLEC2-mediated platelet activation might also be required. To test for platelet-LEC interaction at this site, we examined the left LV junction, where the TD meets the SV. The TD-SV junction was identified in E17.5 embryos by its characteristic location below the clavicle and at the intersection of the internal jugular vein and SV, and by the presence of a bicuspid LVV. As recently described (23), immunostaining of the LVV revealed the presence of $\mathrm{PROX} 1^{+}$endothelial cell nuclei on both sides of the valve, but the LEC-specific surface proteins PDPN and LYVE1 were selectively expressed on the lymphatic side, not the venous side, of this unique valve (Figure 4B). Small platelet aggregates were observed immediately at the LVV, and larger thrombi containing both platelets and erythrocytes were observed along the PROX $1^{+}$LYVE $1^{+}$lymphatic endothelium within the neighboring TD, in a fraction of wild-type animals ( $n=4$ of 14 ; Figure 4C). In contrast, analysis of the TD-SV junction in Clec2 $2^{--}$embryos revealed no platelet aggregates or thrombi, despite the presence of blood filling the TD ( $n=0$ of 4 ; Figure 4D). Notably, LVV structure and size in $\mathrm{Clec}^{-/-}$embryos was indistinguishable from that of wild-type littermates (compare Figure 4, B and C). These observations indicate that platelet-LEC interactions and platelet thrombi form in a CLEC2-dependent manner in the region of the LV junction, despite the presence of the LVV.

$L V$ hemostasis supports valve function to maintain blood-lymphatic separation. The studies described above suggested that CLEC2-mediated platelet activation may function together with the LVV to prevent blood from entering the TD, even in healthy animals with normal valve development and function. To better understand the interaction of these 2 protective mechanisms, we next examined the role of platelets in mice with normal PDPN-CLEC2 signaling, but impaired valve function. PROX1 was strongly expressed on both blood and lymphatic valve endothelium, and Prox $1^{+/-}$mice lacked LVVs (Figure 5A and ref. 23). Integrin $\alpha 9$ is selectively expressed on venous and lymphatic valve endothelium, and Itga9-/mice lack lymphatic valves $(24,25)$. We detected normal-appearing LVVs in Itga9 ${ }^{--}$mice (Figure 5A), but whether they function normally is not known. Histologic analysis of the TD in E17.5 Prox $1^{+/-}$and Itga9 $9^{-/-}$animals revealed the presence of large platelet thrombi both at the LVV and throughout the TD (Figure 5, B-D). In contrast to those in wild-type animals, platelet thrombi in Prox $1^{+/-}$and Itga9 ${ }^{-/-}$animals were both more spatially extensive (i.e., located in more proximal regions of the TD, far from the LV junction; Figure 5D) and more prevalent (i.e., observed in all 6 Itga9 $^{-/-}$animals and all 5 Prox $1^{+/-}$animals examined). In contrast, only a subset of E15-E16 Prox $1^{+/}$animals exhibit bloodfilled lymphatic vessels in the skin (23), while no Itga9-/- animals exhibit this phenotype (24). Thus, LV hemostasis can compensate for loss of LVV and lymphatic valve function to maintain bloodlymphatic separation in vivo.

LV hemostasis involves fibrin formation and platelet aggregation. Our findings revealed an unexpected role for platelet activation in blocking the retrograde entry and passage of venous blood into the lymphatic network. Most previously described platelet responses have been in the setting of arterial shear and vessel injury. In contrast, the terminal TD is predicted to be a site of low flow and low shear, raising the question of whether and to what extent platelet-mediated LV hemostasis uses the same mechanisms as more classic platelet responses. One such mechanism is platelet procoagulant activity that promotes thrombin and fibrin clot formation. Platelet aggregates observed in wild-type animals stained strongly for fibrin (Figure 6A), which indicates that CLEC2-mediated platelet aggregates at the LV junction drive the formation of fibrin-containing thrombi. A second canonical endpoint of platelet activation in the setting of vessel injury is the activation of integrin allbb3 receptors that mediate platelet cross-linking and shear-resistant aggregate formation. To test the role of this mechanism in LV hemostasis, we next analyzed the LV junction of 

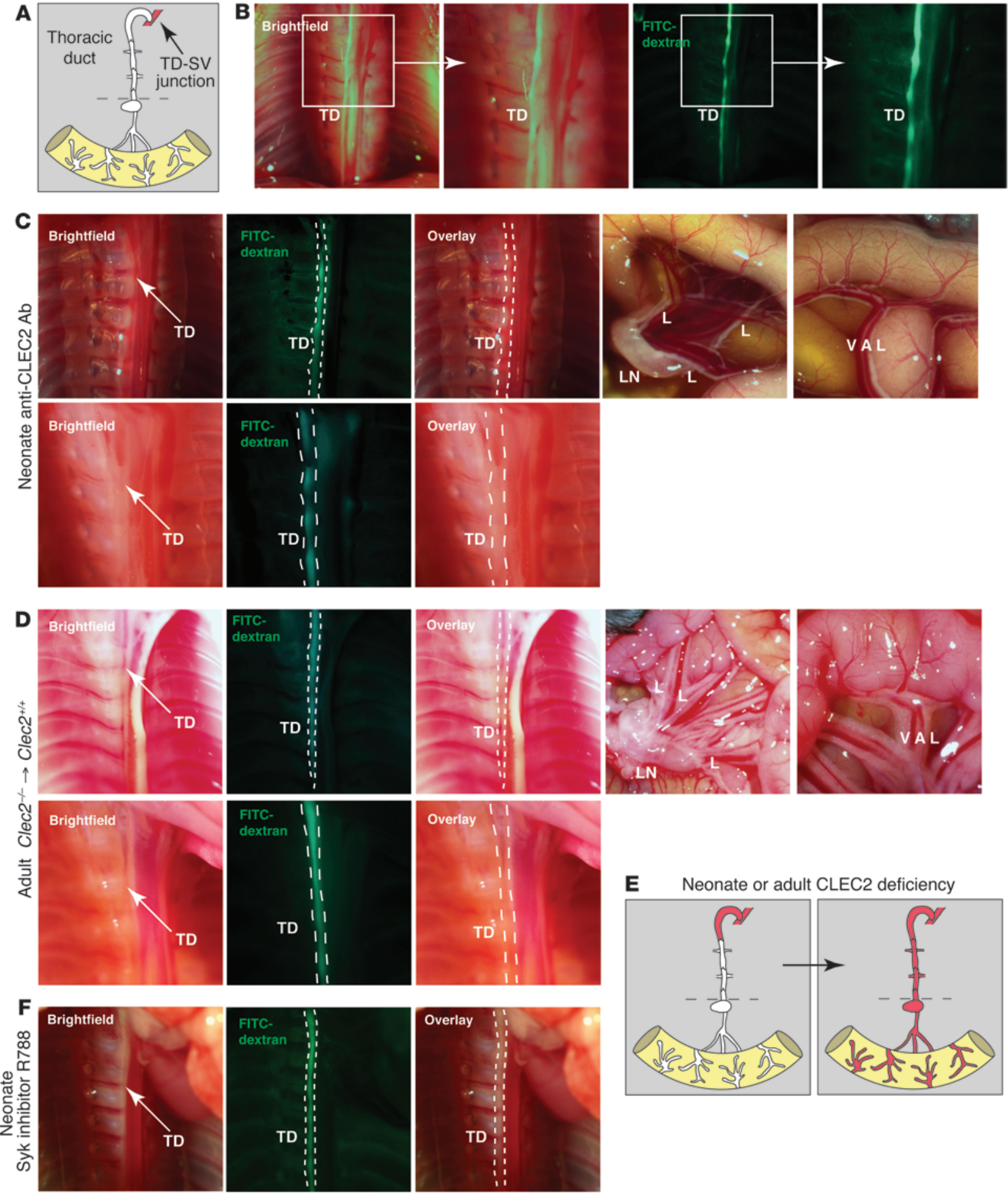

Figure 3

Blood is first observed in the TD after loss of $\mathrm{Clec} 2$ in both neonates and mature animals. (A) Terminal lymphatic network between the intestine and LV junction. Dotted lines denote level of the diaphragm. (B) The TD at P6 was detected adjacent to the vertebral column in the chest by the presence of FITC-dextran after injection into the hindlimb. Boxed regions are shown enlarged at right. (C) Blood was detected in the TD prior to the lymphatics of the mesentery and intestine after loss of CLEC2. Shown are TD (top left) and abdomen (top right) of a P6 wild-type animal injected with anti-CLEC2 antibodies on P1 after FITC-dextran injection. Arrows indicate blood in the TD. Note the absence of blood in lymphatics of the intestine (top) and its presence in the terminal region of the TD near the LV junction (bottom). (D) 12-week-old wild-type mice were lethally irradiated and reconstituted with $\mathrm{Clec2^{-/ }}$ hematopoietic cells, and the TD was imaged 3 weeks later. Arrows indicate blood in the TD. Note the absence of blood in lymphatics of the intestine (top right). Blood was first observed in the terminal region of the TD near the LV junction after reconstitution (bottom). (E) Path of blood from the TD to the lymphatics of the mesentery and intestine after induced CLEC2 deficiency. (F) TD after 8 days of fostamatinib treatment of wild-type mice. Blood was not detected in the TD $(n=6)$. 
A

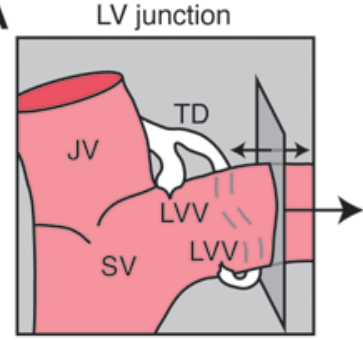

Coronal plane

B

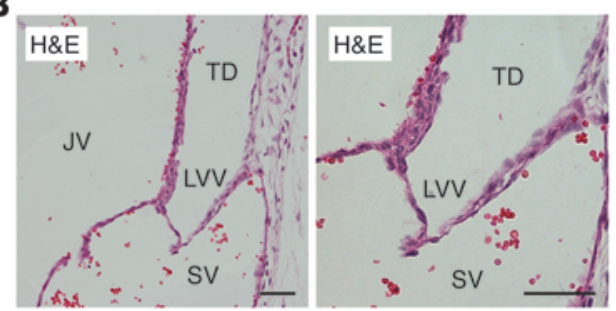

c
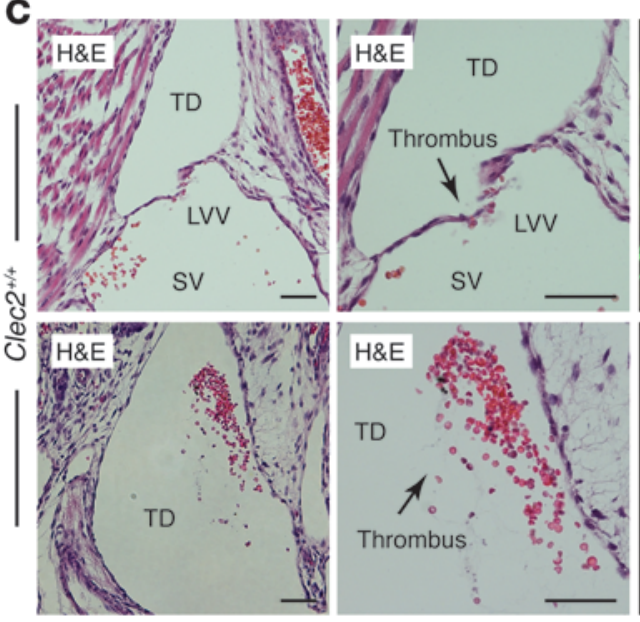

D

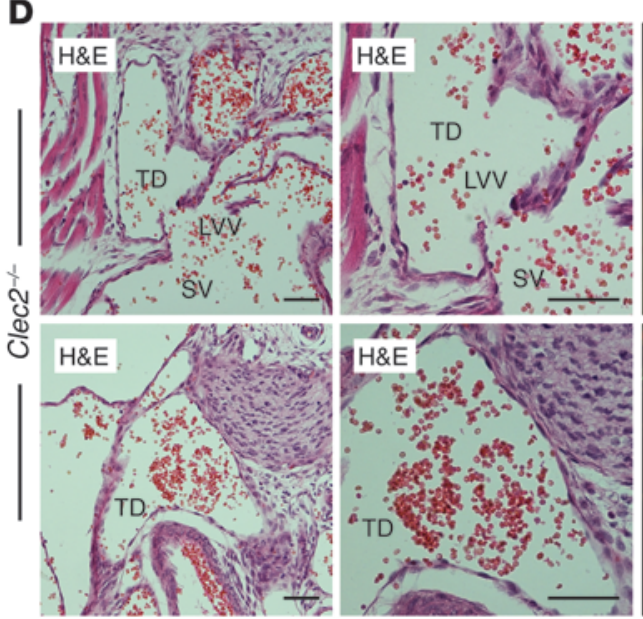

LV juction anatomic variants

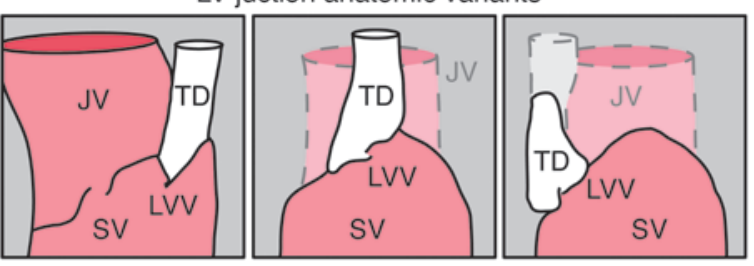

Sagittal plane
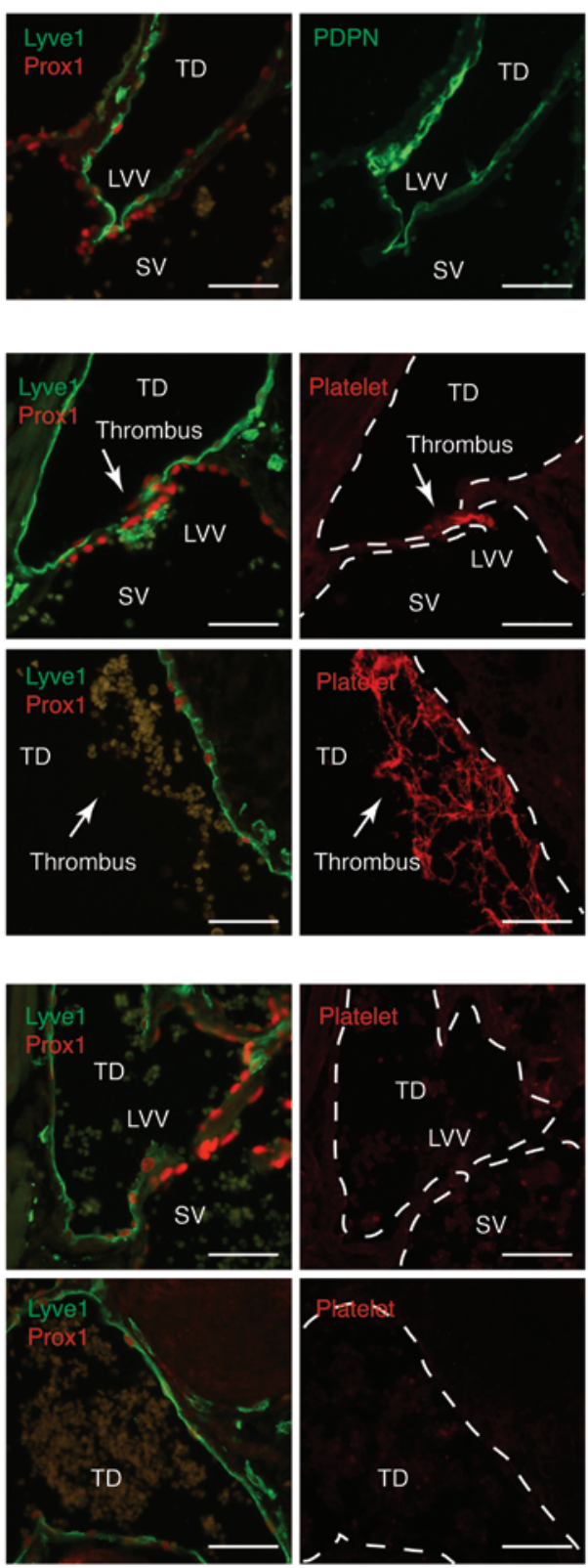

\section{Figure 4}

Platelet thrombi are observed at the LV junction in wild-type mice, but not $\mathrm{Clec2}^{-/-}$mice. (A) LV junction in the coronal and sagittal planes, including some anatomic variants like those described below. (B) LV junction of a wild-type E17.5 embryo in the sagittal plane. PROX1 was detected in endothelial nuclei on both the venous (SV) and lymphatic (TD) sides of the LVV, whereas PDPN and LYVE1 were detected exclusively on the lymphatic side. (C) Platelet thrombi (arrows) were detected at the LVV and adjacent to lymphatic endothelium in the nearby TD in wild-type animals. (D) Blood filled the TD at the LV junction in $\mathrm{Clec}^{-/-}$animals, but no thrombi were observed. Dotted outlines denote vessel walls. JV, jugular vein. Scale bars: $50 \mu \mathrm{m}$.
E17.5 Itgb3 $^{-/-}$embryos. LV thrombi were evident in all 4 Itgb3 ${ }^{-/-}$animals examined (Figure 6B), which indicates that platelet aggregation is not absolutely required for thrombus formation. However, Itgb3-/- animals also exhibited a large amount of blood and thrombi in the TD that was not seen in control littermates (Figure 6C), which suggests that platelet aggregation may function to stabilize thrombi that form in the lymphatic vascular environment. These results identified platelet thrombus formation as the key endpoint of PDPN-CLEC2 signaling and provided further evidence for a hemostatic mechanism of blood-lymphatic separation. 
A

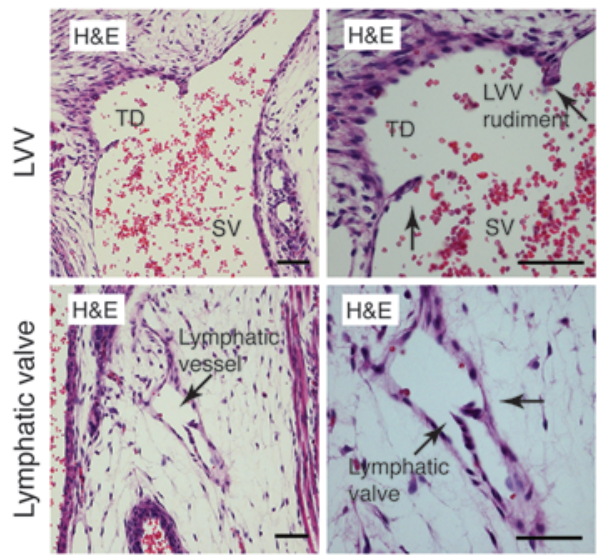

B

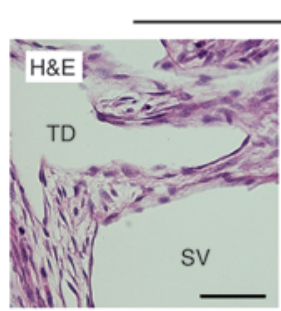

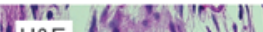

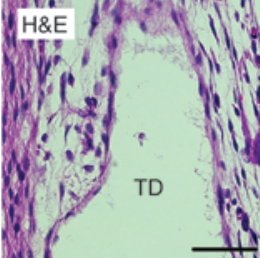

D

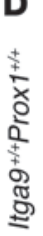
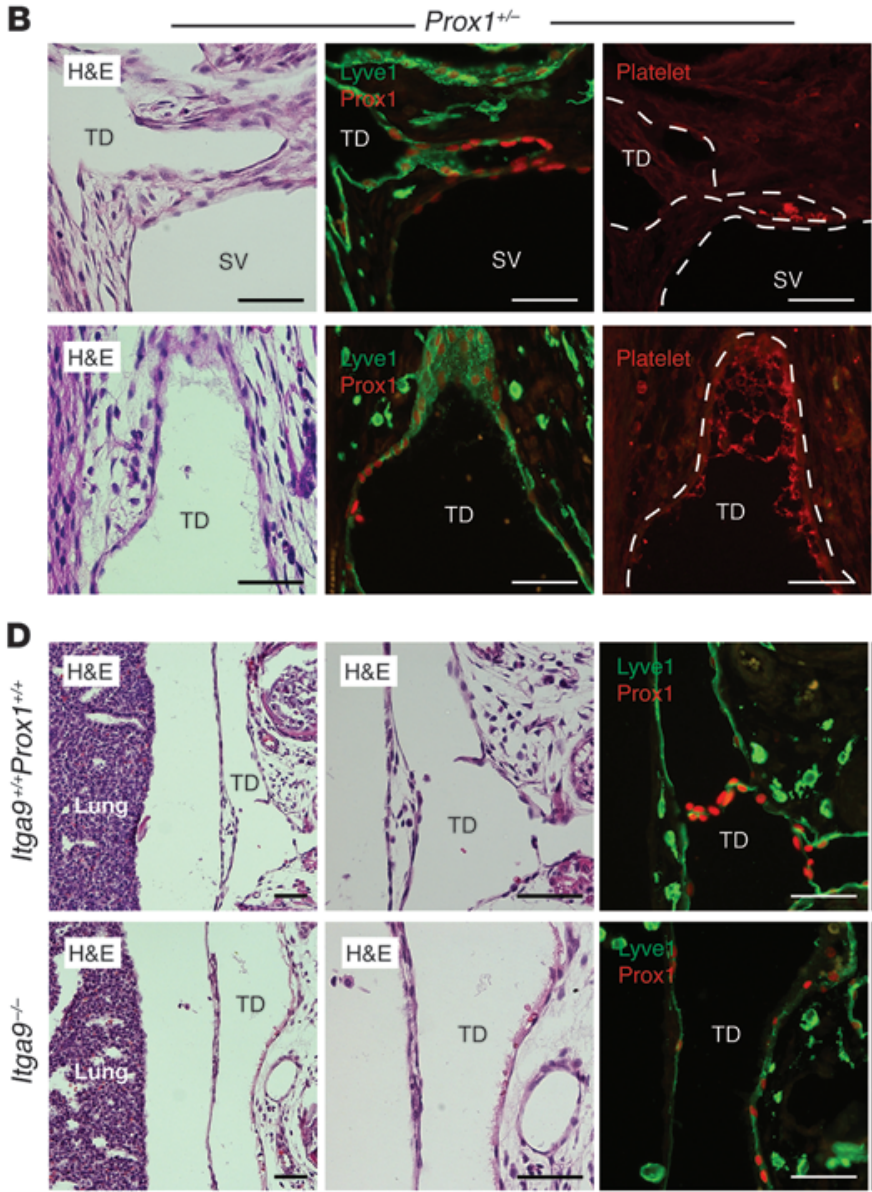
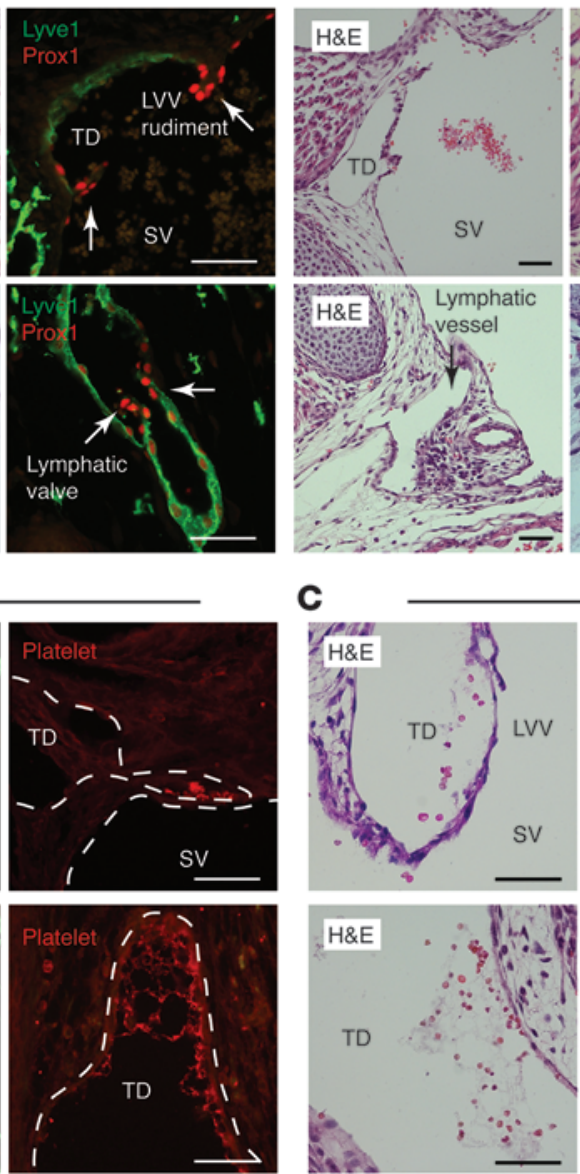

Itga9-1

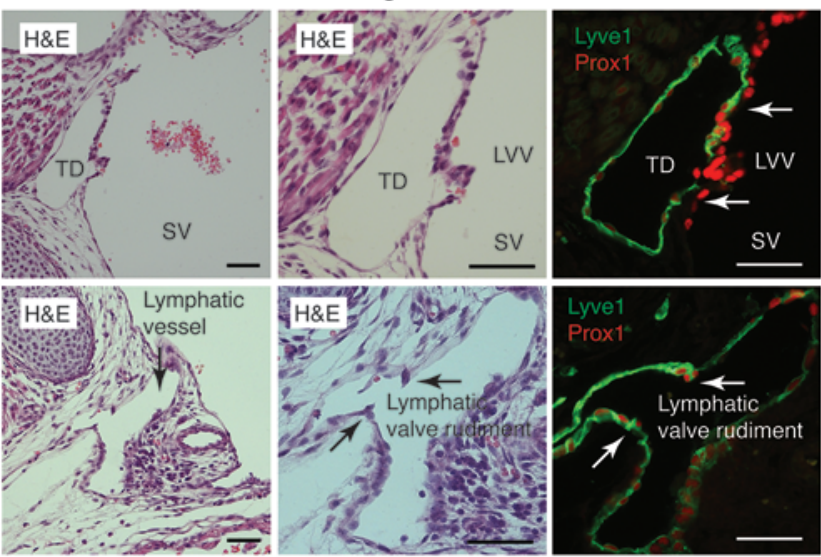

Itga9-
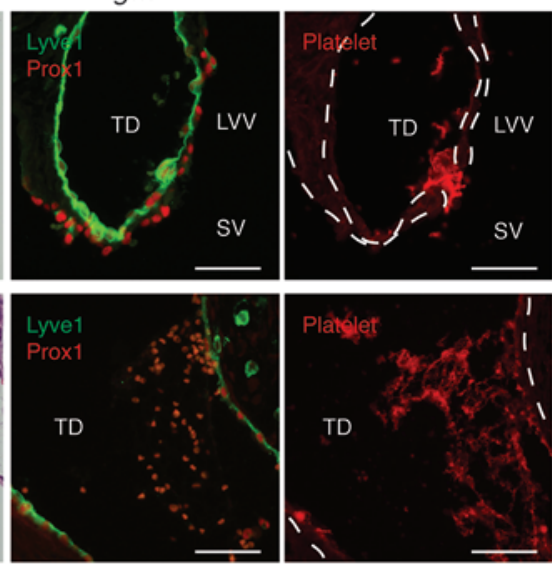

\section{Figure 5}

Large platelet thrombi are observed throughout the TD of Prox1+/- and Itga9-/animals. (A) Representative LVVs and lymphatic valves in Prox $1^{+/-}$and $\operatorname{ltga9}^{-/-}$mice, which had impaired valve development. Valve rudiments are labeled and/or denoted by arrows. (B) Platelet-containing thrombi were observed in the TD adjacent to the SV in E17.5 Prox $1^{+/-}$embryos. (C) Plateletcontaining thrombi were observed next to the LVV and in the TD adjacent to the SV in E17.5 /tga9-/- embryos. (D) Platelet-containing thrombi extended to the proximal TD in the chest of E17.5 Prox1+/- and Itga9-/embryos. Dotted outlines denote vessel walls. Scale bars: $50 \mu \mathrm{m}$. 


\section{A}
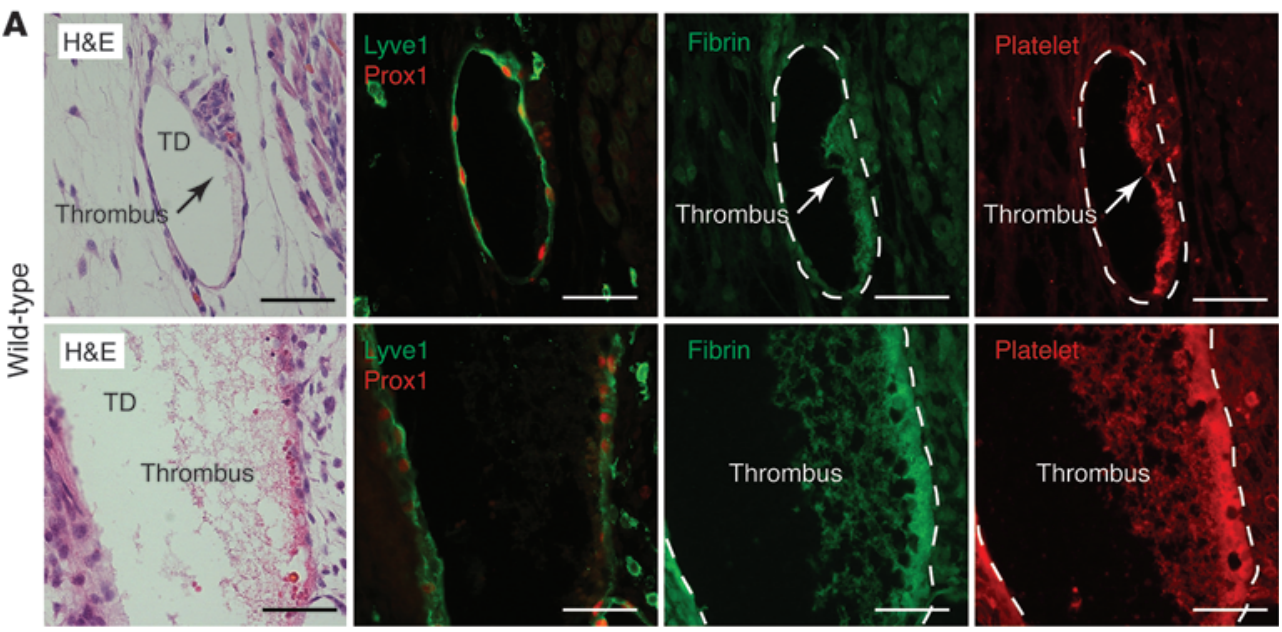

\section{B}
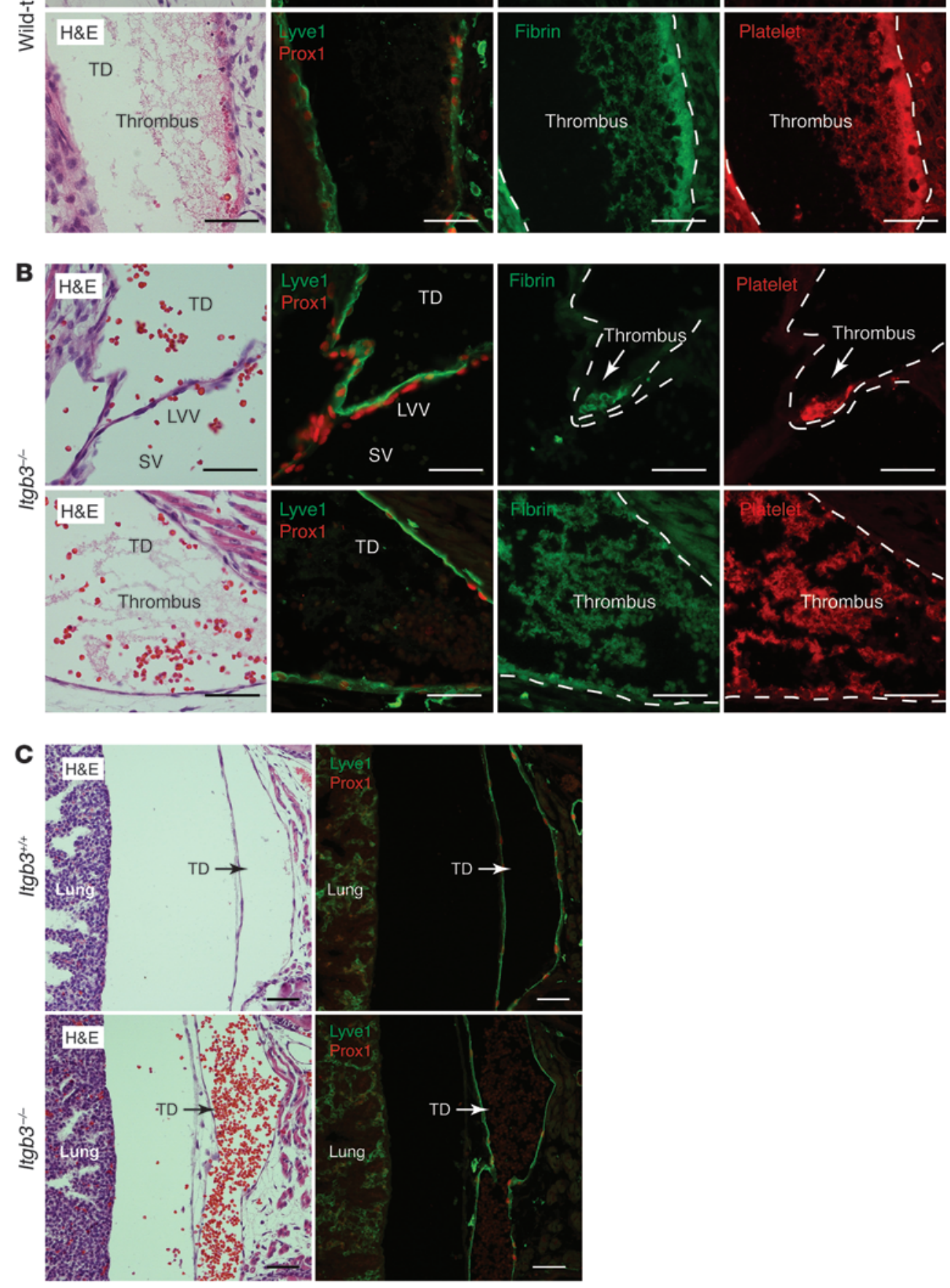

\section{Discussion}

Blood and lymphatic vessels play essential, complementary roles in vivo that require very different structures and function. Blood circulates in a closed vascular network in which flow is driven by

\section{Figure 6}

LV hemostasis involves fibrin generation and platelet aggregation. (A) Platelet thrombi in the terminal TD of wild-type animals contained abundant fibrin. (B) The LV junction and terminal TD of E17.5 Itg b3 $^{-/-}$ embryos contained numerous fibrin+ platelet thrombi. (C) Blood was present in the proximal TD in the chest of $\mathrm{E} 17.5 \mathrm{Itg} \mathrm{b3}^{-/-}$embryos despite thrombus formation. Scale bars: $50 \mu \mathrm{m}$. 

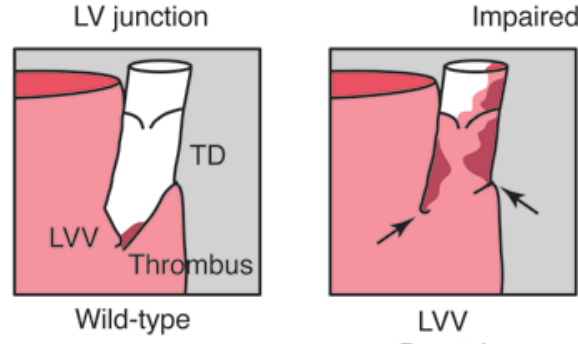

Prox $1^{+1-}$
Impaired valves

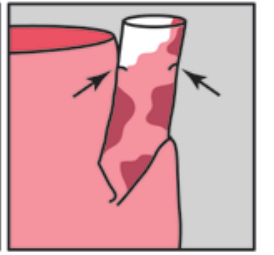

Lymphatic valves Itga9--

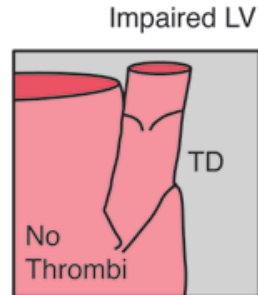

Platelet

PDPN response $\mathrm{Clec}^{-1-}$

\section{Figure 7}

Maintenance of blood-lymphatic separation by LVV function and LV hemostasis. Examination of wild-type and mutant animals revealed the roles of the LVV and CLEC2-mediated LV hemostasis. Left: In healthy wild-type animals, LV hemostasis appears to function intermittently to block the retrograde movement of blood that enters the TD, despite normal LVV function. Middle: Impaired valve function, due either to abnormal LVV formation in Prox $1^{+/-}$animals or to abnormal lymphatic valve formation in Itga9 ${ }^{-/-}$animals, results in more extensive LV hemostasis that prevents backfilling of the lymphatic network with blood. Right: Loss of platelet CLEC2 function results in backfilling of the lymphatic network with blood despite normal LVV function, while loss of platelet aggregation results in a blood-filled TD despite the formation of platelet thrombi.

ultimately drain into the blood at the LV junction, but the open, low-pressure lymphatic system cannot function properly if it is exposed to blood vascular hemodynamic forces. In the present study, we demonstrated that platelet CLEC2 receptor signaling mediated an intervascular hemostasis that is required to safeguard the lymphatic system from these forces and maintain normal lymphatic function throughout life. We found that this LV hemostatic mechanism operates alongside, but is not redundant with, LVVs and lymphatic valves (Figure 7). Our present results define a novel type of platelet-mediated hemostasis and explain the complex vascular phenotypes of mice lacking the PDPN-CLEC2-SYK-SLP-76PLCg2 platelet activation pathway.

Analysis of Syk-deficient mice almost 20 years ago revealed an unexpected phenotype characterized by a "hemorrhagic" appearance in midgestation embryos and fatal chylous ascites in neonates $(18,19)$. Studies in the past decade have established that this phenotype is due to blood-filled lymphatics that arise as a result of loss of platelet activation by the CLEC2 receptor in response to the PDPN ligand expressed on LECs, but not on blood endothelial cells $(4-6,8,26)$. However, precisely when and where platelets interact with lymphatic endothelial PDPN, and how platelet activation prevents blood-lymphatic mixing, has remained mysterious. Our studies demonstrate that the blood-lymphatic mixing phenotypes in postnatal mice lacking this pathway reflect a requirement for platelet interaction with LECs at the LV connection where the TD connects to the SV. Since a primitive LV junction is present by E11.5 (23), it is possible that blood-filled lymphatics in prenatal mice lacking this pathway arise due to the same mechanism. Although it has been suggested that blood-filled lymphatics may arise due to loss of platelet granule contents that regulate lymphangiogenesis $(5,6)$, our findings support a unique intervascular hemostatic mechanism in which CLEC2-activated platelets generate thrombi that prevent blood from entering the lymphatic vascular network at LV junction.

Our findings identify a novel mechanism of platelet-mediated hemostasis that is required for normal lymphatic function and survival. The present paradigm is one that defines the role of platelets primarily by their formation of arterial, shear-resistant aggregates in response to vessel injury and exposure of procoagulant molecules such as tissue factor (27-29). In contrast, our studies reveal an essential mechanism of platelet-mediated hemostasis that operates completely within an uninjured intravascular environment, and under low-flow, low-shear conditions. Our preliminary characterization of this hemostatic pathway revealed that it uses platelet activation to drive procoagulant activity and formation of fibrin-containing thrombi. Consistent with the observation that mice lacking the platelet aIIbb3 integrins do not exhibit blood-filled lymphatics like those lacking CLEC2 signaling (30), thrombus formation in LV hemostasis did not require integrin aIIbb3-mediated platelet aggregation (Figure 6B). However, we did observe impaired LV hemostasis in $\mathrm{Itgb3}^{-/-}$animals (Figure 6C). These findings suggest that multiple known endpoints of platelet activation are likely to participate in LV hemostasis and provide important additional evidence for a hemostatic mechanism of blood-lymphatic separation.

An important paradox raised by our findings is how thrombi formed as a consequence of platelet-LEC interaction can sustain forward lymphatic flow while preventing backward blood flow. While the answer to this question is not yet clear, several possibilities merit discussion. First, while blood circulates at a rate of $5-6 \mathrm{l} / \mathrm{min}$ in the average human, lymph flow is only $3-5 \mathrm{l} / \mathrm{d}$. Thus, thrombi that temporarily occlude the TD and are sufficient to prevent transient backflow of blood into the lymphatic network are not likely to have an immediate effect on lymphatic function. Second, since we observed robust fibrin formation, it is likely that LV thrombi are subject to lysis by fibrinolytic enzymes. Whether rapid clot lysis is more likely to take place in the lymphatic vascular environment (e.g., due to higher fibrinolytic activity in lymph versus plasma, as has been reported previously; refs. 31,32 ) is an interesting possibility that remains to be tested.

The discovery of a specialized platelet hemostatic mechanism to prevent blood from entering the lymphatic system is surprising, given the existence of valves that protect both the LV junction and the TD from backward flow. Why are 2 mechanisms required? Recent genetic studies of vascular development and older physiologic studies of lymphatic function identify several potential reasons for the existence of a second, hemostatic mechanism. Genetic studies in mice revealed that both blood and lymphatic valves mature in late gestation and after birth, time points after formation of the LV junction and the onset of lymphatic function in the 
developing animal $(17,23,25)$. Thus, it is likely that this hemostatic mechanism is initially required to safeguard the lymphatic system during the process of valve development and maturation. This possibility is supported by the observation that Itga9-/- neonates die shortly after birth due to a complete absence of lymphatic valves, but do not exhibit blood-filled lymphatics. However, our finding that blood rapidly entered the TD of adult animals with normal valves after reconstitution with $\mathrm{Clec} 2^{-/-}$hematopoietic cells (Figure $3 \mathrm{G}$ ) revealed that $\mathrm{LV}$ hemostasis was required even after valve development was complete. This ongoing requirement most likely reflects important differences in fluid dynamics between the venous and lymphatic systems that would drive blood into the lymphatic system. Central venous pressure is $5-10 \mathrm{~mm} \mathrm{Hg}$ under resting conditions (33), but is highly dynamic and changes during respiration, with changes in body position and fluid status, and in disease states such as congestive heart failure (CHF). In contrast, lymphatic pressures are typically low, at $1-2 \mathrm{mmHg}$ (34), and elevation of TD pressure beyond $6 \mathrm{~mm} \mathrm{Hg}$ virtually halts lymphatic flow (35). Thus, even small amounts of intermittent backflow of blood might raise lymphatic pressures and obstruct lymphatic flow. Analogous to the role of hemostasis in maintaining blood vessel integrity, $\mathrm{LV}$ hemostasis is probably not a primary means of separating the blood and lymphatic vasculatures in mature animals, but instead most likely functions as a safety mechanism by which to protect lymphatic function.

The discovery of a platelet-mediated hemostatic mechanism required to safeguard the lymphatic system has important clinical implications. A recent ultrasound study of the LV junction in live human patients revealed that elevated central venous pressures due to chronic CHF can be associated with LVV insufficiency, reflux of blood into the TD, and dilatation of the TD (36). These findings are consistent with our findings that mice with deficient valve function exhibited extensive TD thrombus formation (Figure 5) and suggest that the role of platelet-mediated LV hemostasis in safeguarding the lymphatic system might be augmented in patients with CHF. Such patients are frequently treated with antiplatelet drugs to reduce the risk of myocardial infarction and stroke; whether such therapies also compromise lymphatic function and exacerbate respiratory symptoms associated with pulmonary edema is not yet known. A second clinical issue raised by our studies concerns the safety and long-term lymphatic vascular effects of SYK antagonists that have been developed to treat chronic inflammatory disorders such as rheumatoid arthritis $(37,38)$. Genetic studies in mice clearly demonstrate that complete loss of SYK function confers blood-lymphatic mixing in both developing and mature animals as a result of its effects on platelet CLEC2 signaling $(9,19)$. In contrast, we found that pharmacologic loss of SYK function conferred by fostamatinib treatment in mice was not sufficient to confer blood-lymphatic mixing at doses capable of treating a rodent model of arthritis (Figure $3 \mathrm{~F}$ ). These results are encouraging for anti-SYK agents, but whether patients who are also taking more effective antiSYK agents or other antiplatelet drugs and/or have chronically elevated venous pressure (e.g., in association with CHF) will be similarly protected is not yet clear. Understanding the role of this
LV hemostatic pathway in both health and disease is essential for understanding its potential clinical importance.

\section{Methods}

Mouse lines. Clec2 $2^{-/-}$(4), Prox1 $1^{+/-}(39,40)$, and $\operatorname{Itg} a 9^{-/-}$(41) animals have been described previously. $\mathrm{Lta}^{-/}$, $\mathrm{RorC}^{-/-}$, and $\mathrm{Itgb3}^{-/-}$mice were obtained from the Jackson Laboratory. Phenotypic analysis was conducted on

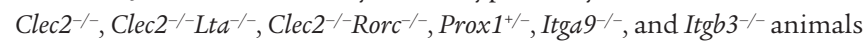
with littermate controls.

Fetal liver transplantation. Fetal livers were isolated from E14.5-E17.5 embryos, passed through a 70- $\mu \mathrm{m}$ cell strainer with DMEM, and injected i.v. into 6- to 8-week-old irradiated recipients (Cesium-137, 11 Gy, Gammacell 40 Exactor; MDS Nordion). Drinking water contained trimethoprim/ sulfamethoxazole antibiotics for 4 weeks after transplantation.

INU1-mediated CLEC2 deficiency. Monoclonal rat anti-mouse CLEC2 antibody (clone INU1, IgG $\operatorname{Ig}_{1}$ in PBS was injected i.p. at a dose of $8 \mu \mathrm{g} / \mathrm{g}$ body weight as described previously (13).

Fostamatinib administration to mice. An aqueous suspension of fostamatinib disodium (R788) at $4.0 \mathrm{mg} / \mathrm{ml}$ was fed orally to neonatal mice every 6 hours at $40 \mathrm{mg} / \mathrm{kg}$ body weight beginning at P1 for 8 days.

Flow cytometry. Whole blood collected into heparinized PBS was immunostained with antibodies to CLEC2 (clone INU1) and GP1b $\alpha$ (clone Xia.G5). Cells were incubated with antibody for 45 minutes, fixed with $5 \%$ formalin for 5 minutes, and stained as usual.

Fluorescent lymph contrast. FITC-dextran (MW 40,000; Sigma-Aldrich) in PBS was injected into the hindlimbs at $10 \mathrm{mg} / \mathrm{ml}$, and lymphatic vessels were imaged 2 hours later.

Histologic analysis. Paraffin-embedded tissue sections were immunostained or H\&E stained. Immunofluorescence was performed with polyclonal anti-mouse antibodies for platelets (Inter-Cell Technologies Inc.), fibrin(ogen) (Santa Cruz Biotechnology), PDPN (R\&D), PROX1 (Abcam), and LYVE-1 (R\&D). Histologic images were acquired with a Nikon Eclipse $80 \mathrm{i}$ microscope using a $\times 20 / 0.50$ or $\times 40 / 0.75$ numeric aperature (NA) dry objective.

Statistics. Kaplan-Meier survival curves were compared using both the logrank (Mantel-Cox) test and the Gehan-Breslow-Wilcoxon test on Prism 5.0 (GraphPad Software). A $P$ value less than 0.05 was considered significant.

Study approval. Animal protocols were approved by the University of Pennsylvania Institutional Animal Care and Use Committee.

\section{Acknowledgments}

We thank Wolfgang Bergmeier, Gary Koretkzy, Skip Brass, Michael Beers, David Artis, and members of the Kahn lab for valuable insights during the course of these studies. This work was supported by NIH grants HL007439 (to P.R. Hess) and HL103432 and by the Leducq Foundation (to M.L. Kahn).

Received for publication April 10, 2013, and accepted in revised form September 26, 2013.

Address correspondence to: Mark L. Kahn, University of Pennsylvania, Translational Research Center, Room 11-123, 3400 Civic Center Boulevard, Building 421, Philadelphia, Pennsylvania 19104, USA. Phone: 215.898.9007; Fax: 215.573.2094; E-mail: markkahn@mail.med.upenn.edu.
1. Tammela T, Alitalo K. Lymphangiogenesis: Molec-
ular mechanisms and future promise. Cell. 2010 ;
140(4):460-476.
2. El Zawahry MD, Sayed NM, El-Awady HM, Abdel-
Latif A, El-Gindy M. A study of the gross, micro- scopic and functional anatomy of the thoracic duct and the lympho-venous junction. Int Surg. 1983; 68(2):135-138.

3. Fu J, et al. Endothelial cell O-glycan deficiency causes blood/lymphatic misconnections and con- sequent fatty liver disease in mice. J Clin Invest. 2008; 118(11):3725-3737.

4. Bertozzi CC, et al. Platelets regulate lymphatic vascular development through CLEC-2-SLP-76 signaling. Blood. 2010;116(4):661-670. 
5. Suzuki-Inoue K, et al. Essential in vivo roles of the C-type lectin receptor CLEC-2: embryonic/neonatal lethality of CLEC-2-deficient mice by blood/ lymphatic misconnections and impaired thrombus formation of CLEC-2-deficient platelets. J Biol Chem. 2010;285(32):24494-24507.

6. Finney BA, et al. CLEC-2 and Syk in the megakaryocytic/platelet lineage are essential for development. Blood. 2012;119(7):1747-1756.

7. Carramolino L, Fuentes J, García-Andrés C, Azcoitia V, Riethmacher D, Torres M. Platelets play an essential role in separating the blood and lymphatic vasculatures during embryonic angiogenesis. Circ Res. 2010;106(7):1197-1201.

8. Abtahian F, et al. Regulation of blood and lymphatic vascular separation by signaling proteins SLP-76 and Syk. Science. 2003;299(5604):247-251.

9. Kiefer $F$, et al. The Syk protein tyrosine kinase is essential for Fcgamma receptor signaling in macrophages and neutrophils. Mol Cell Biol. 1998;18(7):4209-4220.

10. Osada M, et al. Platelet activation receptor CLEC-2 regulates blood/lymphatic vessel separation by inhibiting proliferation, migration, and tube formation of lymphatic endothelial cells. J Biol Chem. 2012;287(26):22241-22252.

11. Bertozzi CC, Hess PR, Kahn ML. Platelets: covert regulators of lymphatic development. Arterioscler Thromb Vasc Biol. 2010;30(12):2368-2371.

12. Uhrin P, et al. Novel function for blood platelets and podoplanin in developmental separation of blood and lymphatic circulation. Blood. 2010; 115(19):3997-4005.

13. May F, et al. CLEC-2 is an essential platelet-activating receptor in hemostasis and thrombosis. Blood. 2009;114(16):3464-3472.

14. Abtahian F, et al. Evidence for the requirement of ITAM domains but not SLP-76/Gads interaction for integrin signaling in hematopoietic cells. Mol Cell Biol. 2006;26(18):6936-6949.

15. Neumann B, Luz A, Pfeffer K, Holzmann B. Defective Peyer's patch organogenesis in mice lacking the $55-\mathrm{kD}$ receptor for tumor necrosis factor. J Exp Med. 1996;184(1):259-264.

16. Kurebayashi S, et al. Retinoid-related orphan receptor gamma (RORgamma) is essential for lymphoid organogenesis and controls apoptosis during thymopoiesis. Proc Natl Acad Sci U S A. 2000; 97(18):10132-10137.

17. Sabine A, et al. Mechanotransduction, PROX1, and FOXC2 cooperate to control connexin 37 and calcineurin during lymphatic-valve formation. Dev Cell. 2012;22(2):430-445.

18. Cheng AM, Rowley B, Pao W, Hayday A, Bolen JB, Pawson T. Syk tyrosine kinase required for mouse viability and B-cell development. Nature. 1995; 378(6554):303-306

19. Turner M, et al. Perinatal lethality and blocked B-cell development in mice lacking the tyrosine kinase Syk. Nature. 1995;378(6554):298-302.

20. Pine PR, et al. Inflammation and bone erosion are suppressed in models of rheumatoid arthritis following treatment with a novel Syk inhibitor. Clin Immunol. 2007;124(3):244-257.

21. Young RM, et al. Mouse models of non-Hodgkin lymphoma reveal Syk as an important therapeutic target. Blood. 2009;113(11):2508-2516.

22. Braselmann S, et al. R406, an orally available spleen tyrosine kinase inhibitor blocks fc receptor signaling and reduces immune complex-mediated inflammation. J Pharmacol Exp Ther. 2006;319(3):998-1008.

23. Srinivasan RS, and Oliver G. Prox1 dosage controls the number of lymphatic endothelial cell progenitors and the formation of the lymphovenous valves. Genes Dev. 2011;25(20):2187-2197.

24. Bazigou E, et al. Integrin-alpha9 is required for fibronectin matrix assembly during lymphatic valve morphogenesis. Dev Cell. 2009;17(2):175-186.

25 . Bazigou E, et al. Genes regulating lymphangiogenesis control venous valve formation and maintenance in mice. J Clin Invest. 2011;121(8):2984-2992.

26. Suzuki-Inoue K, et al. A novel Syk-dependent mechanism of platelet activation by the C-type lectin receptor CLEC-2. Blood. 2006;107(2):542-549.

27. Davì G, Patrono C. Platelet activation and atherothrombosis. N Engl J Med. 2007;357(24):2482-2494.

28. Nesbitt WS, et al. A shear gradient-dependent platelet aggregation mechanism drives thrombus formation. Nat Med. 2009;15(6):665-673.
29. Jackson SP. The growing complexity of platelet aggregation. Blood. 2007;109(12):5087-5095.

30. Hodivala-Dilke KM, et al. $\beta 3$-Integrin-deficient mice are a model for Glanzmann thrombasthenia showing placental defects and reduced survival. J Clin Invest. 1999;103(2):229-238.

31. Lippi G, Favaloro EJ, Cervellin G. Hemostatic properties of the lymph: relationships with occlusion and thrombosis. Semin Thromb Hemost. 2012; $38(2): 213-221$.

32. Chrobák L, Bartos V, Brzek V, Hnízdová D. Coagulation properties of human thoracic duct lymph. Am J Med Sci. 1967;253(1):69-75.

33. Guyton AC, Hall JE. Textbook of Medical Physiology. 9th ed. Philadelphia, Pennsylvania, USA: W.B. Saunders Company; 2000.

34. Browse NL, Lord RS, Taylor A. Pressure waves and gradients in the canine thoracic duct. J Physiol. 1971;213(3):507-524.

35. Elk JR, Laine GA. Pressure within the thoracic duct modulates lymph composition. Microvasc Res. 1990;39(3):315-321.

36. Seeger M, et al. Terminal part of thoracic duct: high-resolution US imaging. Radiology. 2009; 252(3):897-904

37. Weinblatt ME, et al. Effects of fostamatinib (R788), an oral spleen tyrosine kinase inhibitor, on healthrelated quality of life in patients with active rheumatoid arthritis: analyses of patient-reported outcomes from a randomized, double-blind, placebo-controlled trial. J Rheumatol. 2013;40(4):369-378.

38. Hoellenriegel J, et al. Selective, novel spleen tyrosine kinase (Syk) inhibitors suppress chronic lymphocytic leukemia B-cell activation and migration. Leukemia. 2012;26(7):1576-1583.

39. Wigle JT, Chowdhury K, Gruss P, Oliver G. Prox1 function is crucial for mouse lens-fibre elongation. Nat Genet. 1999;21(3):318-322.

40. Wigle JT, Oliver G. Prox1 function is required for the development of the murine lymphatic system. Cell. 1999;98(6):769-778.

41. Huang XZ, et al. Fatal bilateral chylothorax in mice lacking the integrin alpha9beta1. Mol Cell Biol. 2000;20(14):5208-5215. 